\title{
Sub-30 nm Plasmonic Nanostructures by Soft UV Nanoimprint Lithography
}

\author{
Grégory Barbillon
}

Additional information is available at the end of the chapter

http://dx.doi.org/10.5772/56119

\section{Introduction}

The capability to fabricate nanostructures of high density and high resolution over large areas is important point for fundamental and applied research as subwavelength optical nanostructures, optoelectronics and biosensors [1-5]. Various lithographic techniques such as focused ion beam lithography [6] and electron beam lithography [7, 8] are mainly used to pattern sub-100 nm structures on large surfaces. However, these two techniques are slow to obtain these surfaces and their equipments are expensive. Moreover, charge effect on insulating surface can alter the regularity of the pattern shape. Thus, these techniques will not be suitable for a mass production. In addition, alternative methods emerged in the past decades, and are not very expensive and fast to realize high density nanostructures. Moreover, these methods offer a better compatibility for biology and chemical applications $[9,10]$. One of these recent techniques is the soft UV Nanoimprint Lithography, which is very promising for the periodic nanostructures fabrication with a high density and high resolution on large surfaces for a reasonable cost $[11,12]$. However, a limiting factor of UV-NIL is the resolution of the fabricated molds [13, 14]. In soft UV-NIL, cast molding processes are used for flexible mold realization. An advantage of the soft UV-NIL technique is the obtaining of a great patterns homogeneity on a large zone. This chapter proposes to present in details the soft UV-NIL and its use for the fabrication of sub-30 nm plasmonic structure on large area.

\section{Principle \& fabrication process of soft UV nanoimprint lithography}

\subsection{Principle of UV-NIL}

Soft UV-NIL has two fundamental steps and is illustrated in figure 1. Firstly, a UV transparent mold with nanostructures on its surface is pressed into a UV sensitive resist. The UV sensitive resist, which is liquid at room temperature, is typically spin coated on 
the substrate. The UV transparent stamp is deposited on the substrate with a low pressure between 0 and 1 bar [15], at room temperature. Next, the soft stamp is released. The first step duplicates the nanopattern of the transparent mold in the UV sensitive resist. The second step is the removal of residual layer of UV sensitive resist. This step is realized by anisotropic etching such as Reactive Ion Etching (RIE) in order to obtain the desired patterns into the UV resist. During the nanoimprint step, the resist is cured by a UV source (for example, a simple UV lamp or other available systems).

The main advantages of soft UV-NIL are the transparent flexible stamp and a low viscosity UV-curable resist. The replication of the flexible stamps is typically obtained by molding and curing a polymer from a 3D template. The UV transparent flexible stamp fabrication is mainly realized with poly(dimethylsiloxane) PDMS [16-18]. PDMS offers a good chemical stability and a high optical transparency. Moreover, the deformation risk of the soft stamp is minimized with the use of low viscosity UV-curable resists, which permits 3D patterning at low pressure without any heating cycles.

\section{Press Stamp}

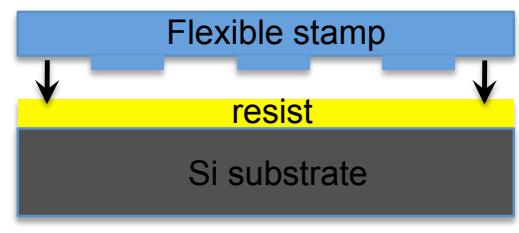

\section{Release Stamp}

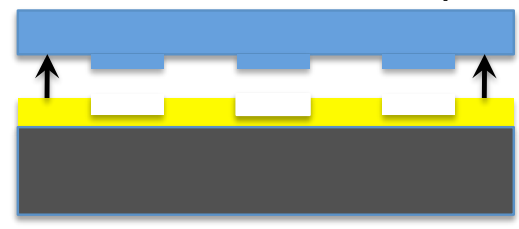

\section{RIE process}

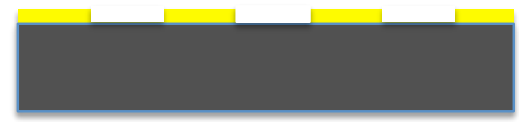

Figure 1. Scheme of UV nanoimprint lithography process: (1) Imprint with the stamp (+ curing with UV light), (2) Stamp withdrawal and (3) Removal of the residual layer with RIE process.

\subsection{Master mold fabrication}

The master mold used to fabricate flexible stamp is made using the electron beam lithography (EBL). EBL technique allows an excellent accuracy, a very high resolution, and an capability to pattern a large variety of geometries. In the example presented here for the Si master mold fabrication, a PMMA layer of $100 \mathrm{~nm}$ (PolyMethylMethAcrylate A2 resist: 950 PMMA A2, MicroChem Corp.) is deposited by spin-coating on Si substrate and baked at $170^{\circ} \mathrm{C}$ during $30 \mathrm{~min}$. An EBL system (Leica EBPG5000+) is used to expose PMMA A2, employing 
an accelerating voltage of $100 \mathrm{kV}$. Then, the sample is developed in a methylisobutylketone (MIBK)/isopropanol (IPA) solution at room temperature for $35 \mathrm{~s}$ followed by a rinsing of $10 \mathrm{~s}$ in IPA and thoroughly dried with $\mathrm{N}_{2}$ gas. Next, the patterns designed in PMMA are transferred into the silicon substrate via a suitable RIE process. The RIE conditions are: $10 \mathrm{sccm}$ for $\mathrm{O}_{2}, 45 \mathrm{sccm}$ for $\mathrm{SF}_{6}$ with $\mathrm{P}=30 \mathrm{~W}$, a pressure of $50 \mathrm{mTorr}$ and an autopolarization voltage of $85 \mathrm{~V}[15,17]$. Then, the PMMA mask is removed with a lift-off process in trichloroethylene at $80^{\circ} \mathrm{C}$. Next, the Si master mold surface is treated with HF and $\mathrm{H}_{2} \mathrm{O}_{2}$ in order to obtain a $\mathrm{SiO}_{2}$ thin surface layer, then modified with an anti-sticking layer (TMCS: TriMethylChloroSilane) for decreasing the surface energy ( $\mathrm{Si}+\mathrm{TMCS}=28.9$ $\mathrm{mN} / \mathrm{m}$ ) in order to easy remove the PDMS molds [17, 19, 20]. In figure 2, SEM images of nanostructures obtained on a Si substrate are presented.
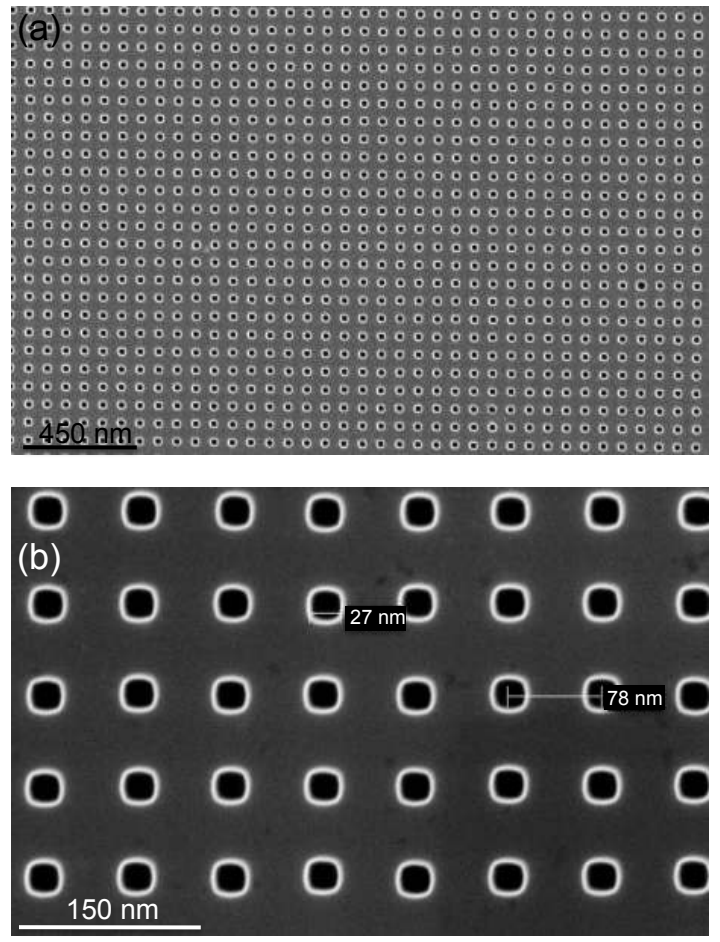

Figure 2. SEM images of Si master mold designed by EBL: nanoholes of diameter $\sim 27 \mathrm{~nm}$ and $\sim 78 \mathrm{~nm}$ of periodicity. (a) zone of some $\mu \mathrm{m}^{2}$ and (b) zoom of the previous zone.

For the master mold, the dimensions of obtained nanoholes are $\sim 27 \mathrm{~nm}$ of diameter and $\sim$ $78 \mathrm{~nm}$ of periodicity on a zone of $1 \mathrm{~cm}^{2}$. The programmed dimensions for the electron beam lithography are $25 \mathrm{~nm}$ for the diameter and $75 \mathrm{~nm}$ for the pitch. Consequently, the dimensions obtained experimentally are in good agreement with those programmed by taking into account the errors of measurements, which could be observed on the nanostructure dimensions with SEM. 


\subsection{Flexible UV-transparent stamp fabrication}

\subsubsection{Polymeric materials for stamp fabrication}

An advantage of polymeric materials for the replication of original master mold in the nanoimprint process is the low cost of fabrication compared to EBL. With a single and expensive master mold, a large number of polymeric stamps can be replicate and use in the nanoimprint process. Moreover, the excellent flexibility of the elastomeric material offers a good contact between the stamp and the substrate on large areas at low pressures (tens of bars) and on non-planar substrates. Various polymeric materials have been used for stamp fabrication as cross-linked novolak based epoxy resin [21], polycarbonate resins [22], fluoropolymer materials and tetrafluoroethylene (PTFE) [23]. In addition, poly(dimethylsiloxanes) (PDMS) have very interesting properties as a stamp elastomer. The first of these properties is a conformal adhesion of the stamp with the substrate on large areas without any external pressure.

Indeed, PDMS has other attractive properties: (1) its flexibility, which allows a good accuracy of relief shapes replication in the fabrication of the patterning elements, (2) its low Young's modulus $(750 \mathrm{KPa})$ [24] and its low surface energy which allows conformal contact with surface without applied pressure and non-destructive release from designed structures [25], (3) its good optical transparency to a UV light source [26], and (4) its commercial availability in bulk quantities at low cost. The standard PDMS has some advantages, however a number of properties inherent to PDMS limits its performances in the soft UV-NIL. First, the Young's modulus of standard PDMS is low and can limit the fabrication of high density patterns at a sub-100 nm scale due to the collapse of structures. Second, the surface energy $(\sim 20 \mathrm{mN} / \mathrm{m})$ of PDMS is not low enough and that does not make it possible the duplication of profiles with a high fidelity. Moreover, the high elasticity and thermal expansion can lead to deformations and distortions during the fabrication process. In general, long range deformations can be avoided by using a thin glass backplane which preserves a global flexibility. In addition, short range deformations can be avoided only by increasing the elastic modulus of PDMS (see paragraph 2.3.2).

\subsubsection{Standard PDMS stamp fabrication process}

Standard PDMS stamps are mainly realized with a mixture of two commercial PDMS components: (10:1) PDMS RTV 615 siloxane oligomer and RTV 615 cross-linking oligomers (General Electric). This mixture is deposited then casted on the Si master mold and degassed in a dessicator. Standard PDMS is cured at $60^{\circ} \mathrm{C}$ for $24 \mathrm{~h}$ in order to reduce roughness and to avoid a build up of tension due to thermal shrinkage. If longer curing times and higher temperatures are used, then the elastic modulus and hardness of the polymer are increased (up to $\mathrm{x} 2$ ). However, a higher roughness and deformations can be observed. The stamps are cooled to room temperature, thoroughly peeled off from the master mold and treated with the TriMethylChloroSilane (TMCS) anti-sticking layer in order to reduce the low PDMS surface energy. These stamps are not suitable for the replication of sub- $100 \mathrm{~nm}$ structures (or with a high aspect ratio) due to the low elastic modulus of PDMS. To solve this problem of low Young's modulus, a modified PDMS called hard-PDMS was already developed. 


\subsubsection{Bilayer hard-PDMS/PDMS stamp fabrication process}

To increase the resolution and fidelity of structures, the mechanical properties of the soft stamp need to be improved. Odom et al. [28] developed a bilayer stamp of hard-PDMS and standard PDMS, which presents, as advantages, a rigid layer to obtain a high resolution pattern transfer and an elastic support for obtaining a conformal contact even at a low imprint pressure. The hard-PDMS has an attractive property: a lower viscosity of its prepolymer in comparison to standard PDMS. The hard-PDMS prepolymer viscosity is obtained by decreasing of the chain length during its preparation. Thus, the accuracy of replication is improved especially for high-density and small patterns. Another groups [23, 29] also studied the viscosity reduction of the prepolymer for an good replication of the master mold. In this case, the PDMS prepolymer viscosity was decreased with the introduction of a solvent in the mixture. This solvent used with an excessive amount of modulator allows to delay the cross-linking.

The figure 3 represents the fabrication process of the bilayer hard-PDMS/PDMS stamp. The hard-PDMS is a specific thermocured siloxane polymer based on copolymers Vinylmethylsiloxane-Dimethylsiloxane (VDT301) and Methyl-hydrosilane-Dimethylsiloxane (HMS-301) from ABCR, respectively, $34 \mathrm{~g}$ and $11 \mathrm{~g}$ [27]. In addition, before degassing the mixture with a mixing machine we add $50 \mu \mathrm{L}$ of platinum catalyst, and $0.5 \% \mathrm{w} / \mathrm{w}$ modulator tetramethyl-tetravinyl cyclotetrasiloxane from FLUKA to the mixture [30]. Then, the hard-PDMS is spin coated on the silicon master mold and the used thickness is mainly 5-8 $\mu \mathrm{m}$ and supported by a standard PDMS layer $(\sim 1.5 \mathrm{~mm})$ (see figure 3 ). The standard PDMS layer keeps a good flexibility and adaptation on the spin coated wafer during imprint transfer [31]. Then, the bilayer stamp is placed on a glass carrier. The standard PDMS (RTV 615) with its curing agent are mixed before deposit on the thin hard layer PDMS (H-PDMS). Finally, the sample is cured at $60^{\circ} \mathrm{C}$ during 24 hours and treated with a TriMethylChloroSilane (TMCS) anti-sticking layer.

For the chosen example of nanostructures, the bilayer stamp is very suitable. Indeed, the obtained nanodots dimensions are $\sim 28 \mathrm{~nm}$ of diameter, the periodicity of $\sim 78 \mathrm{~nm}$, and the height of $\sim 60 \mathrm{~nm}$. The figure 4 represents an AFM image of the hard-PDMS/PDMS stamp.

\subsection{Soft UV-NIL process}

\subsubsection{Optimization of pressure and decreasing of possible deformations for imprint step}

In order to realize the best pattern replication, the control of possible deformations of the replicated pattern is a very important point. Indeed, the PDMS stamp flexibility allows to obtain a conformal adhesion with the substrate at low pressure, during the imprint process. Nevertheless, structures deformations with a high aspect ratio can occur when the applied pressure increases due to the low Young's modulus. In the case where these deformations cannot be avoided, their control and reduction are keypoints and depend on the wished application. A study of the resolution was made by some groups. KarlSuss $\mathrm{GmbH}$ has studied the replication of nanoholes (340 nm of diameter) in AMONIL resist. They obtained a diameter of $340 \mathrm{~nm} \pm 5 \%$, and a period uniformity of $2 \mathrm{~nm}$ over a 6 inch area [32]. During the imprint step, the resist flow depends strongly on the applied pressure and determines the accuracy on the dimensions of the imprinted nanostructures. In order to reduce local distortions, the pressure of the imprint step must be minimized to obtain a good depth of 
1. Master mold designed by EBL in PMMA

+ Transfer of patterns in Si by RIE $\left(\mathrm{O}_{2}+\mathrm{SF}_{6}\right)$

+ Lift-off of PMMA

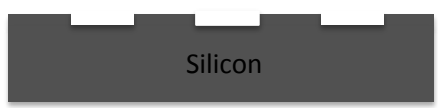

2. Hard-PDMS spin coated (thickness 5-8 $\mu \mathrm{m}$ )

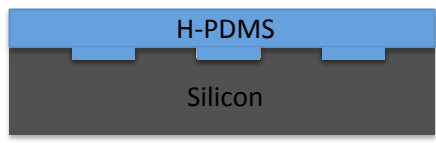

3. PDMS Casting $(\approx 1.5 \mathrm{~mm})$ and curing at $60^{\circ} \mathrm{C}$ during $24 \mathrm{~h}$

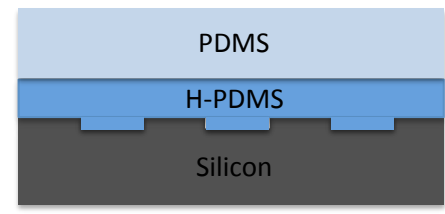

4. Bilayer Hard-PDMS/PDMS Stamp Release

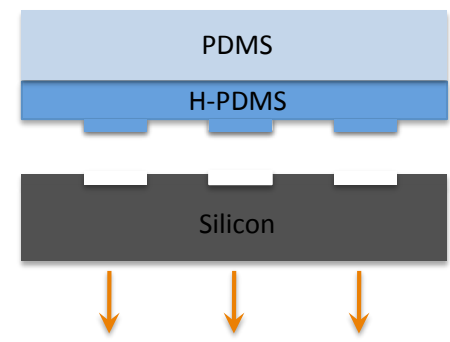

Figure 3. Principle scheme of the fabrication process of the hard-PDMS/PDMS stamp.

the resist in the stamp nanostructures. Cattoni et al. [33] demonstrated that the pressure could be reduced to 0.7 bar (thus that Shi et al. [19] ) and combined with a UV exposure of $10 \min \left(\lambda=365 \mathrm{~nm}\right.$, dose of $\left.2 \mathrm{~J} / \mathrm{cm}^{2}\right)$, a high quality of nanostructures shape was obtained.

\subsubsection{Optimization of the thickness of the resist residual layer}

Another keypoint of the NIL process is the removal of the residual layer of the resist. In Thermal-NIL and standard UV-NIL, a rigid mold and a high pressure are used, and a thin residual layer of resist is mainly left between the mold protrusions and the substrate. It acts as a soft cushion layer that prevents direct impact of these fragile nanostructures and the substrate. The residual layer is typically withdrawn by RIE. The RIE step can strongly affect the initial shape and size of nanostructures. In addition, in the soft UV-NIL, which uses flexible stamps, the residual layer can be reduced by adapting the original resist thickness to the height (or depth following the desired pattern) of the stamp pattern. Several groups demonstrated the adaptation of the initial resist thickness to the height or depth of the 


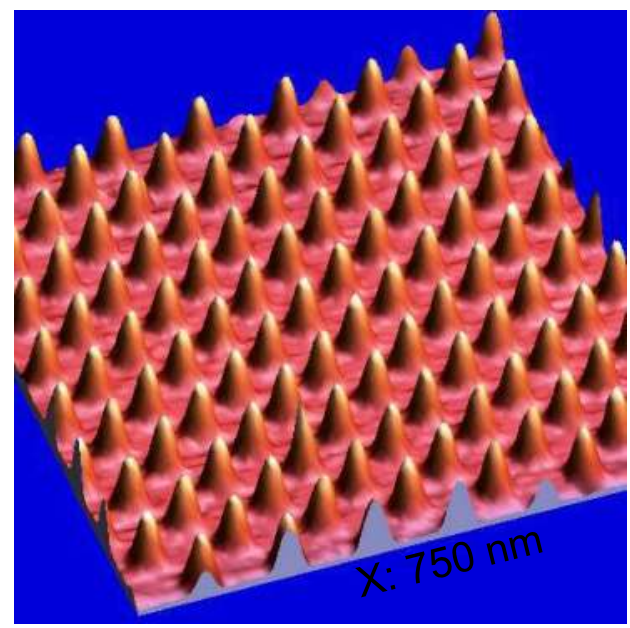

Figure 4. AFM image of the dots in the bilayer hard-PDMS/PDMS stamp (periodicity: $\sim 78 \mathrm{~nm}$, diameter (FWHM): $\sim 28 \mathrm{~nm}$, and height: $\sim 60 \mathrm{~nm}$ ).

stamp pattern in order to decrease the residual thickness of resist layer. One of these groups conducted a study on the AMONIL resist and they obtained a structure depth of $170 \mathrm{~nm}$ and a residual layer of $36 \mathrm{~nm}$ [32]. Thus, a very small thickness of the residual AMONIL layer was observed and allowed a good replication.

\subsection{Soft UV-NIL for sub-30 $\mathrm{nm}$ plasmonic nanostructures fabrication}

\subsubsection{Soft UV-NIL in AMONIL}

Various UV-sensitive resists as the NXR 2010 and the AMONIL are available. These two resists exhibit good performance for resolution and etching resistance. The AMONIL resist was used for its low cost compared to the NXR 2010 resist, and its excellent time of conservation. AMONIL resist is a mixture of organic and inorganic compounds having a surface energy of $39.5 \mathrm{mN} / \mathrm{m}$. AMONIL MMS10 from AMO GmbH is used and spin coated on the top of a Ge/PMMA A2 bilayer (10 nm/100 nm thick, respectively), which allows the AMONIL lift-off after curing (see figure 5(a)). The Ge layer is used to improve the selectivity of the former one over the PMMA layer [20]. An AMONIL thickness of $70 \mathrm{~nm}$ is chosen in order to minimize the residual thickness of AMONIL. Then, the imprint process is performed in AMONIL with UV exposure at $365 \mathrm{~nm}$ wavelength for $10 \mathrm{~min}$ and a pressure of 0.7 bar. All these parameters were optimized for the fabrication of nanostructures, which use the bilayer hard-PDMS/PDMS stamp obtained from Si master mold. The figure 5(b) represents the imprint in AMONIL. The dimensions obtained for nanoholes imprinted in AMONIL are $\sim 28 \mathrm{~nm}$ of diameter and $\sim 78 \mathrm{~nm}$ of periodicity and these values are in good agreement with the dimensions of nanoholes of Si master mold.

\subsubsection{Plasmonic nanodisks fabrication}

Firstly, the residual AMONIL thickness in the ground of the nanoholes must be removed by a suitable RIE process. For the removal of the residual layer, the etch gases used for RIE 
1. Imprint in AMONIL resist with a UV lamp $(\lambda=365 \mathrm{~nm})$ during $10 \mathrm{~min}$ and $\mathrm{P}=0.7$ bar

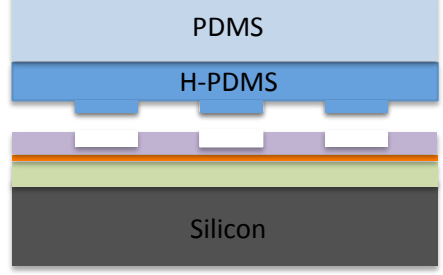

2. RIE of residual trilayer

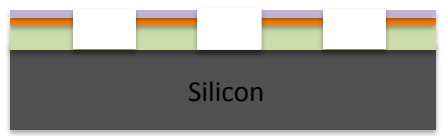

3. Au Evaporation \& Lift-off of PMMA

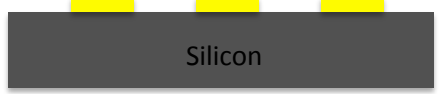

(a)

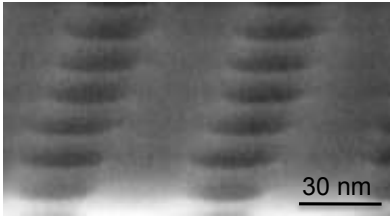

(b)

Gases for RIE of the trilayer:

- AMONIL: $\mathrm{O}_{2}$ and $\mathrm{CHF}_{3}$

- $\mathrm{Ge}: \mathrm{SF}_{6}$

- PMMA: $\mathrm{O}_{2}$

Figure 5. (a) Scheme of the trilayer soft UV-NIL process, and (b) SEM image of imprint in AMONIL with bilayer hard-PDMS/PDMS stamp.

are $\mathrm{O}_{2}$ and $\mathrm{CHF}_{3}$. Ge is removed by RIE using $\mathrm{SF}_{6}$ [34]. For the removal of the PMMA A2, the gas used for RIE is $\mathrm{O}_{2}$. A good selectivity between PMMA and AMONIL is obtained $[15,17]$. The next step is to evaporate a gold thin layer $(30 \mathrm{~nm})$ in order to realize the plasmonic nanodisks. Previously, an adhesion layer $(\mathrm{Cr})$ for gold is evaporated $(3-5 \mathrm{~nm})$. Then, a lift-off in acetone is used to remove the PMMA underlayer (+ AMONIL/Ge) in order to obtain the sub-30 nm plasmonic nanodisks. The figure 6 presents the results obtained with the bilayer hard-PDMS/PDMS stamp in AMONIL. We observe that the dimensions of plasmonic nanodisks are in good agreement with the dimensions obtained with the imprint in AMONIL. Then, the plasmonic nanostructures can be used as Localized Surface Plasmon Resonance biosensors [35].

\section{Conclusion}

In this chapter, we have demonstrated the fabrication of sub-30 $\mathrm{nm}$ plasmonic nanostructures with the soft UV-NIL technique. The soft UV-NIL is composed by three separate steps: the fabrication of the master mold, the replication of the flexible hard-PDMS/PDMS stamp from the Si master mold, and the imprinting process by using the bilayer hard-PDMS/PDMS stamp. All these steps are very important in order to obtain a very good quality of the final result, in terms of resolution and line edge roughness of the nanostructures. A master mold fabrication process based on EBL is presented in details. Then, the replication of the soft polymeric stamp, based on a composite hard-PDMS/PDMS bilayer, is presented. The ability 


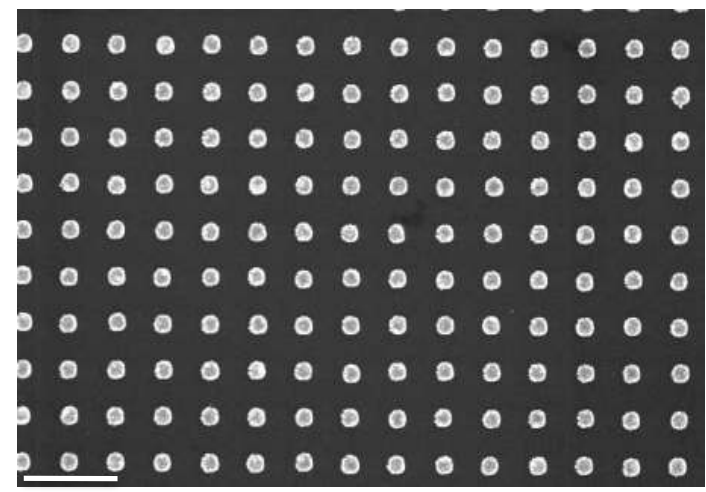

(a)

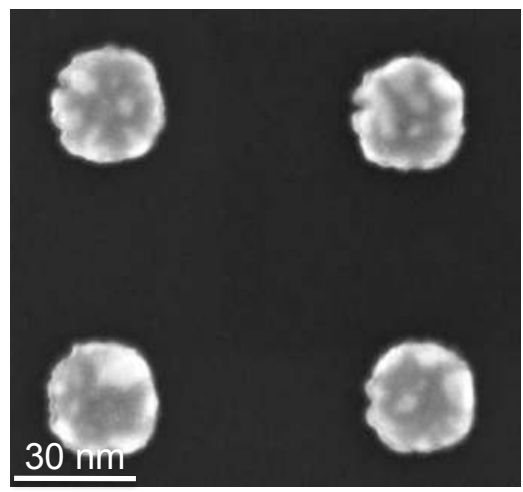

(b)

Figure 6. SEM images of plasmonic nanodisks: (a) a zone of some $\mu \mathrm{m}^{2}$ (scale bar $=150 \mathrm{~nm}$ ), (b) diameter $\sim 28 \mathrm{~nm}$, and periodicity $\sim 78 \mathrm{~nm}$.

of soft UV-NIL to replicate sub-30 nm nanostructures with high homogeneity at the whole pattern surface is demonstrated. To conclude, we present an example of a fabrication by soft UV-NIL on large area $\left(1 \mathrm{~cm}^{2}\right)$ of plasmonic nanostructures with potential applications in biosensors and photonics. Finally, we believe that the soft UV-NIL technique will play quickly an important role as a powerful and versatile tool for the nanostructures fabrication.

\section{Author details}

Grégory Barbillon ${ }^{1,2, \star}$

* Address all correspondence to: gregory.barbillon@laposte.net

1 Laboratory of Photonics and Nanostructures CNRS UPR 20, Marcoussis, France

2 Laboratory of Lasers Physics CNRS UMR 7538, University Paris 13, Villetaneuse, France

\section{References}

[1] Li M, Tan H, Wang J, Chou S.Y (2003) Large area direct nanoimprinting of $\mathrm{SiO}_{2}-\mathrm{TiO}_{2}$ gel gratings for optical applications. J. Vac. Sci. Technol. B 21: 660-663.

[2] Chegel V, Lucas B, Guo J, Lopatynskyi A, Lopatynska O, Poperenko L (2009) Detection of biomolecules using optoelectronic biosensor based on localized surface plasmon resonance. Nanoimprint lithography approach. Semiconductor Physics, Quantum Electronics \& Optoelectronics 12 91-97.

[3] Jensen T.R, Duval M.L, Kelly K.L, Lazarides A.A, Schatz G.C, Van Duyne R.P (1999) Nanosphere Lithography : Effect of the External Dielectric Medium on the Surface Plasmon Resonance Spectrum of a Periodic Array of Silver Nanoparticles. J. Phys. Chem. B 103: 9846-9853. 
[4] Barbillon G, Bijeon J.L, Lérondel G, Plain J, Royer P (2008) Detection of chemical molecules with integrated plasmonic glass nanotips. Surface Science 602: L119-L122.

[5] Faure A.C, Barbillon G, Ou M.G, Ledoux G, Tillement O, Roux S, Fabregue D, Descamps A, Bijeon J.L, Marquette C.A, Billotey C, Jamois C, Benyatou T, Perriat $\mathrm{P}$ (2008) Core/Shell nanoparticles for multiple biological detection with enhanced sensitivity and kinetics. Nanotechnology 19: 485103.

[6] Jiang X, Ji L, Chang A, Leung K.N (2003) Resolution improvement for a maskless microion beam reduction lithography system. J. Vac. Sci. Technol. B 21: 2724-2727.

[7] Vieu C, Carcenac F, Pépin A, Chen Y, Mejias M, Lebib A, Manin-Ferlazzo L, Couraud L, Launois H (2000) Electron beam lithography: resolution limits and applications. Applied Surface Science 164: 111-117.

[8] Barbillon G, Bijeon J.L, Plain J, Royer P (2009) Sensitive detection of biological species through localized surface plasmon resonance on gold nanodisks. Thin Solid Films 517: 2997-3000.

[9] Gates B.D, Xu Q, Stewart M, Ryan D, Wilson C.G, Whitesides G.M (2005) New Approaches to Nanofabrication: Molding, Printing, and Other Techniques. Chemical Reviews 105: 1171-1196.

[10] Krauss P.R, Chou S.Y (1997) Nano-compact disks with 400 Gbit/in ${ }^{2}$ storage density fabricated using nanoimprint lithography and real with proximal probe. Appl. Phys. Lett. 71: 3174-3176.

[11] Chou S.Y, Krauss P.R, Renstorm P.J (1996) Imprint lithography with 25-Nanometer resolution. Science 272: 85-87.

[12] Austin M.D, Ge H, Wu W, Li M, Yu Z, Wasserman D, Lyon S.A, Chou S.Y (2004) Fabrication of $5 \mathrm{~nm}$ linewidth and $14 \mathrm{~nm}$ pitch features by nanoimprint lithography. Appl. Phys. Lett. 84: 5299-5301.

[13] Jung G.Y, Johnston-Halperin E, Wu W, Yu Z, Wang S.Y, Tong W.M, Li Z, Green J.E, Sheriff B.A, Boukai A, Bunimovich Y, Heath J.R, Stanley Williams R (2006) Circuit Fabrication at $17 \mathrm{~nm}$ Half-Pitch by Nanoimprint Lithography. Nano Lett. 6: 351-354.

[14] Austin M.D, Zhang W, Ge H, Wasserman D, Lyon S.A, Chou S.Y (2005) 6 nm half-pitch lines and $0.04 \mu \mathrm{m}^{2}$ static random access memory patterns by nanoimprint lithography. Nanotechnology 16: 1058.

[15] Hamouda F, Barbillon G, Held S, Agnus G, Gogol P, Maroutian T, Scheuring S, Bartenlian B (2009) Nanoholes by soft UV nanoimprint lithography applied to study of membrane proteins. Microelectron. Eng. 86: 583-585.

[16] Hamouda F, Barbillon G, Gaucher F, Bartenlian B (2010) Sub-200 nm gap electrodes by soft UV nanoimprint lithography using polydimethylsiloxane mold without external pressure. J. Vac. Sci. Technol. B 28: 82-85. 
[17] Barbillon G, Hamouda F, Held S, Gogol P, Bartenlian B (2010) Gold nanoparticles by soft UV nanoimprint lithography coupled to a lift-off process for plasmonic sensing of antibodies. Microelectron. Eng. 87: 1001-1004.

[18] Hamouda F, Sahaf H, Held S, Barbillon G, Gogol P, Moyen E, Aassime A, Moreau J, Canva M, Lourtioz J.M, Hanbücken M, Bartenlian B (2011) Large area nanopatterning by combined anodic aluminum oxide and soft UV-NIL technologies for applications in biology. Microelectron. Eng. 88: 2444-2446.

[19] Shi J, Chen J, Decanini D, Chen Y, Haghiri-Gosnet A.M (2009) Fabrication of metallic nanocavities by soft UV nanoimprint lithography. Microelectron. Eng. 86: 596-599.

[20] Chen J, Shi J, Decanini D, Cambril E, Chen Y, Haghiri-Gosnet A.M (2009) Gold nanohole arrays for biochemical sensing fabricated by soft UV nanoimprint lithography. Microelectron. Eng. 86: 632-635.

[21] Pfeiffer K, Fink M, Ahrens G, Gruetzner G, Reuther F, Seekamp J, Zankovych S, Torres C.S, Maximov I, Beck M, Graczyk M, Montelius L, Schulz H, Scheer H.C, Steingrueber F (2002) Polymer stamps for nanoimprinting. Microelectron. Eng. 61-62: 393-398.

[22] Posognano D, D'Amone S, Gigli G, Cingolani R (2004) Rigid organic molds for nanoimprint lithography by replica of high glass transition temperature polymers. J. Vac. Sci. Technol. B 22: 1759-1763.

[23] Kang H, Lee J, Park J, Lee H.H (2006) An improved method of preparing composite poly(dimethylsiloxane) moulds. Nanotechnology 17: 197-200.

[24] Bender M, Plachteka U, Ran J, Fuchs A, Vratzov B, Kurz H, Glinsner T, Lindner F (2004) High resolution lithography with PDMS molds. J. Vac. Sci. Technol. B 22: 3229-3232.

[25] Hsia K.J, Huang Y, Menard E, Park J.U, Zhou W, Rogers J, Fulton J.M (2005) Collapse of stamps for soft lithography due to interfacial adhesion. Appl. Phys. Lett. 86: 154106.

[26] Schmid H, Biebuyck H, Michel B, Martin O.J.M (1998) Light-coupling masks for lensless, sub-wavelength optical lithography. Appl. Phys. Lett. 72: 2379.

[27] Choi D.G, Yu H.K, Yang S.M (2004) 2D nano/micro hybrid patterning using soft/block copolymer lithography. Mater. Sci. Eng. C 24: 213-216.

[28] Odom T.W, Love J.C, Wolfe D.B, Paul K.E, Whitesides G.M (2002) Improved pattern transfer in soft lithography using composite stamps. Langmuir 18: 5314-5320.

[29] Koo N, Bender M, Plachetka U, Fuchs A, Wahlbrink, T, Bolten J, Kurz H (2007) Improved mold fabrication for the definition of high quality nanopatterns by soft UV-nanoimprint lithography using diluted PDMS material. Microelectron. Eng. 84: 904-908.

[30] Schmid H, Michel B (2000) Siloxane Polymers for High-Resolution, High-Accuracy Soft Lithography. Macromolecules 33: 3042-3049. 
[31] Plachteka U, Bender M, Fuchs A, Vratzov B, Glinsner T, Lindner F, Kurz H (2005), Wafer scale patterning by soft UV-Nanoimprint Lithography. Microelectron. Eng. 73/74: 167-171.

[32] Ji R, Hornung M, Verschuuren M.A, van de Laar R, van Eekelen J, Plachetka U, Moeller M, Moormann C (2010) UV enhanced substrate conformal imprint lithography (UV-SCIL) technique for photonic crystals patterning in LED manufacturing. Microelectron. Eng. 87: 963-967.

[33] Cattoni A, Cambril E, Decanini D, Faini G, Haghiri-Gosnet A.M (2010) Soft UV-NIL at $20 \mathrm{~nm}$ scale using flexible bi-layer stamp casted on HSQ master mold. Microelectron. Eng. 87: 1015-1018.

[34] Chen J, Shi J, Cattoni A, Decanini D, Liu Z, Chen Y, Haghiri-Gosnet A.M (2010) A versatile pattern inversion process based on thermal and soft UV nanoimprint lithography techniques. Microelectron. Eng. 87: 899-903.

[35] Barbillon G (2011) Soft UV Nanoimprint Lithography: A Tool to Design Plasmonic Nanobiosensors. In: Kostovski G, editor. Advances in Unconventional Lithography. Rijeka: InTech. pp. 3-14. 